\title{
CRISPRs provide broad and robust protection to oral microbial flora of gingival health against bacteriophage challenge
}

\section{Dear Editor,}

Clustered regularly interspaced short palindromic repeats (CRISPR), which are widely present in prokaryotic genomes (Grissa et al., 2007), belong to a family of DNA sequences characterized as short direct repeats (DR) separated by spacers (Jansen et al., 2002). CRISPR and CRISPR-associated (cas) genes are involved in resistance against exogenous sequences, and recognition of infected bacteriophages depends on the sequence similarity between spacers and targeted phage DNA segments (Barrangou et al., 2007). CRISPRs exhibited diversity in different microbial communities, and that may be the response to different phage infections (Tyson and Banfield, 2008). Dynamic change of spacers in CRIPSR reflects altered resistance to viruses (Andersson and Banfield, 2008), which may play important roles in shaping the structure and driving the evolution of microbial communities. Consequently, in addition to a tool designed for genome editing (Ghorbal et al., 2014), CRISPRs are widely used to demonstrate the dynamic changes of bacteria and phage communities.

In human microbial communities, a study involving more than 700 datasets of metagenomic sequencing reported that only a few spacers were shared among different body sites or individuals although known CRISPRs can be found in most human oral microbiome (Rho et al., 2012). A study on oral microbiome also found that less than $2 \%$ spacers were shared between healthy people as a result of different people may encounter different virus population (Pride et al., 2011). Comparison between oral CRISPR sequences and virome further indicated that viruses and CRISPR diverged significantly among individuals and a large proportion of spacers were specific to each individual and time point (Pride et al., 2012). Another study on CRISPR suggested that spacers from oral bacteria were associated with oral viral ecology (Robles-Sikisaka et al., 2013). These results reveal that CRISPRs were under pressure of dynamic change of viruses in oral environment. Despite the potential effect on oral microbial ecology, little attention was paid to the comparison between CRISPRs under disease and health status until now. As oral cavity is a very complicated environment with diverse bacterial communities and periodontitis is a common disease closely related to microbial flora disorders (Belda-Ferre et al., 2012), we focused on the comparison of CRISPRs of bacterial communities under different periodontal status (in periodontal health and in periodontitis) in this study.

To characterize the CRISPR compositions under different periodontal status and the relationship between healthy and periodontitis patients, we recruited 9 human subjects suffered from periodontitis and 9 health controls. They were selected from mature non-smoking females among 30 60 years old without any other systemic disease. Periodontal status of these volunteers was clinical monitored at six sites per tooth by a periodontist. Probing depth and attachment loss were taken as the main classification criteria. Chronic periodontitis was selected whose periodontal pockets $\geq 4 \mathrm{~mm}$ and attachment loss $\geq 6 \mathrm{~mm}$ at more than 4 tooth sites. Periodontal health had no probing depth $>2 \mathrm{~mm}$ or attachment loss $>2 \mathrm{~mm}$ at any site. Dental plaques were individually collected at least $2 \mathrm{~h}$ after eating and $6 \mathrm{~h}$ after tooth brushing, and DNA was extracted and then was disrupted into fragments with $\sim 180 \mathrm{bp}$ in length. To assess the composition of the microbial communities, they were sequenced to $2 \times 100$ bp paired-end (PE) reads by an Illumina HiSeq 2000 sequencing instrument. In total, we got 176,931,096 reads for 18 samples (Table S1). Previous studies always assemble the reads to contigs to identify CRIPSR arrays, but part of the reads will be omitted in the assembly. To fulfill all the information of the reads, we identified DRs and spacers directly from raw reads by Crass 0.3 .12 (Skennerton et al., 2013) which is on the basis of the distinctive structure of CRISPR. By this step we got 844 DR and 24,841 spacer sequences. The length of DRs and spacers mostly distributed from $30 \mathrm{bp}$ to $40 \mathrm{bp}$ (Fig. 1A). To classify these CRISPR elements, DRs and spacers were respectively aligned to bacteria and phage genomes in NCBI non-redundant (NR) database.

Accordingly, DRs were assigned to 68 bacterial genera (Table S2), with 48 in periodontal health $(\mathrm{PH})$ and 59 in periodontal disease (PD). Spacers were assigned to 444 phage species (444 in PH and 411 in PD, Table S3). Though 
more DRs were found in $\mathrm{PD}, \mathrm{PH}$ possesses 33 more spacers than PD (Fig. 1B). Moreover, PH had more spacercontained reads in 386 of 444 phage species. This result suggests that microbiome from healthy people had a wider spectrum in resisting the invasion of phages than patients suffered from periodontitis. When the oral microbial flora of patients suffered from chronic periodontitis encountered bacteriophages corresponding to the distinctive spacers hold only by healthy people, it is easier to be attacked and might not maintain a stable bacterial community, and microbiota disequilibrium was exactly the reason causing or increasing the susceptibility of periodontal diseases (Curtis et al., 2011). As expected, DRs in PD showed slightly higher ShannonWiener diversities than in $\mathrm{PH}$ (Fig. 1C) and the spacer composition exhibit significantly higher diversities in $\mathrm{PH}$ (Mann-Whitney $U$ test $P$-value $=0.0002$, Fig. 1D) on the contrary. For Bray-Curtis distance, both DRs $(P$-value $=$ $0.0001)$ and spacers $(P$-value $<0.0001)$ have remarkably higher values in patients (Fig. $1 \mathrm{E}$ and $1 \mathrm{~F}$ ). Principle components analysis (PCA) also indicated CRISPR composition distinguished the periodontal status of the oral cavities (Figs. S1 and S2). PH samples had similar composition of DRs and spacers, and thus $\mathrm{PH}$ may have more stable microbial CRIPSRs with a large variety of spacers which can

A
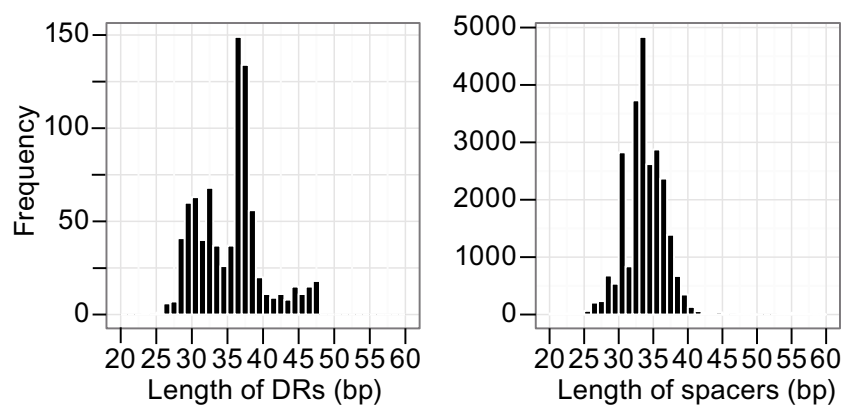

B

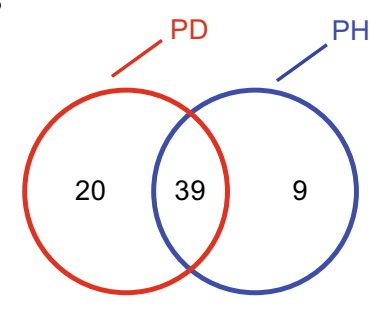

CRISPR DRs

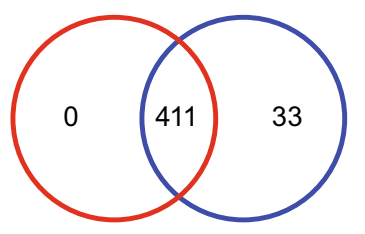

CRISPR spacers
Figure 2. Evenness of the CRISPR DRs and spacers in PD and PH samples. (A) Top 30 most abundant bacterial genera of DR source. Area of each bubble represented the relative abundance (by dividing total reads numbers of each sample and then normalized to 100,000 ) of DRs for each sample (column) and each genus (row). Red color represented PD $(n=9)$ and blue color represented the $\mathrm{PH}$ ( $n=9)$ samples. (B) The most variegated spacers (classified to phage species by BLASTX) between the $\mathrm{PD}$ and $\mathrm{PH}$ groups $(n=9)$. Scales on $\mathrm{x}$-axis represent relative abundance..

defend the invasion from bacteriophages and subsequently preserve the dynamic balance of the whole microbial community.

To characterize the subjects that play a role of defense, we ranked the DRs based on the relative abundance of DRsassigned genera. The relative abundance of each genus was calculated by dividing total reads numbers of each sample and then normalized to 100,000 . Top 30 most abundant genera of DR source were shown in the bubble chart (Fig. 2A), which accounted for $98.8 \%$ of the entire DRs. Common bacteria of human oral cavities such as
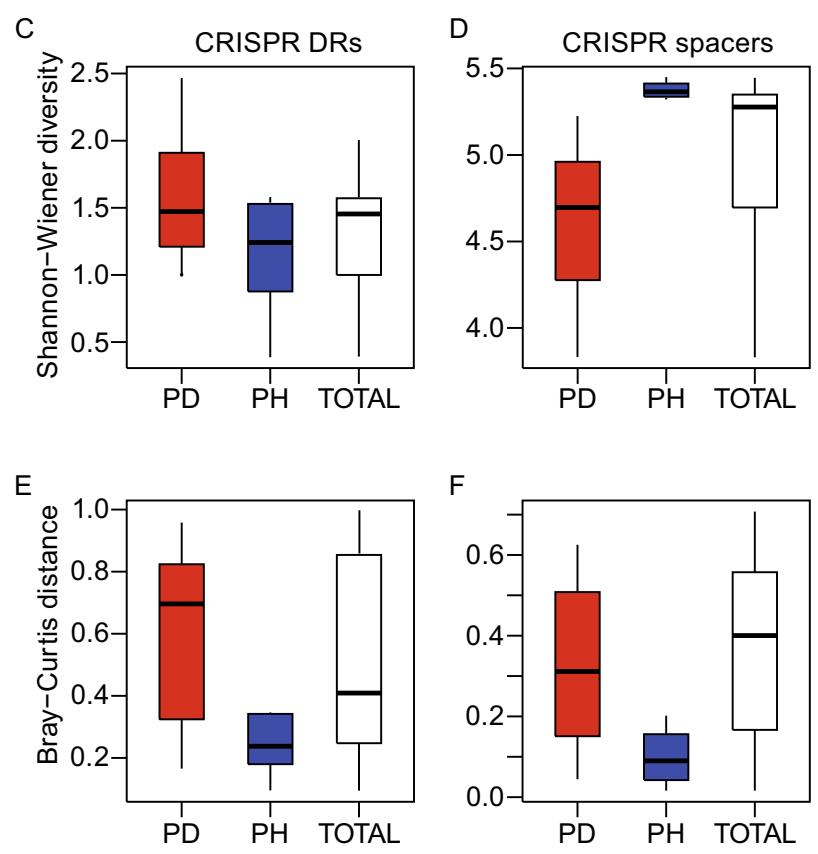

Figure 1. The composition of the CRISPRs in samples collected from patients with chronic periodontitis (PD) and people with healthy gingiva (PH). (A) The distribution of the length of DRs (left panel) and spacers (right panel) identified from metagenomic sequencing data. (B) Numbers of DRs (left panel) and spacers (right panel) in PD (red circle) and PH (blue circle). (C) Shannon-Wiener diversities of the DRs in PD (red box), PH (blue box) and all (white box). (D) Shannon-Wiener diversities of the spacers. (E) Bray-Curtis diversities of the DRs. (F) Bray-Curtis diversities of the spacers. The boxes represent the interquartile range between the first and third quartiles. The whiskers denote the lowest and highest values within the interquartile ranges of the first and third quartiles. The thick lines inside the boxes represent the medians.. 
A

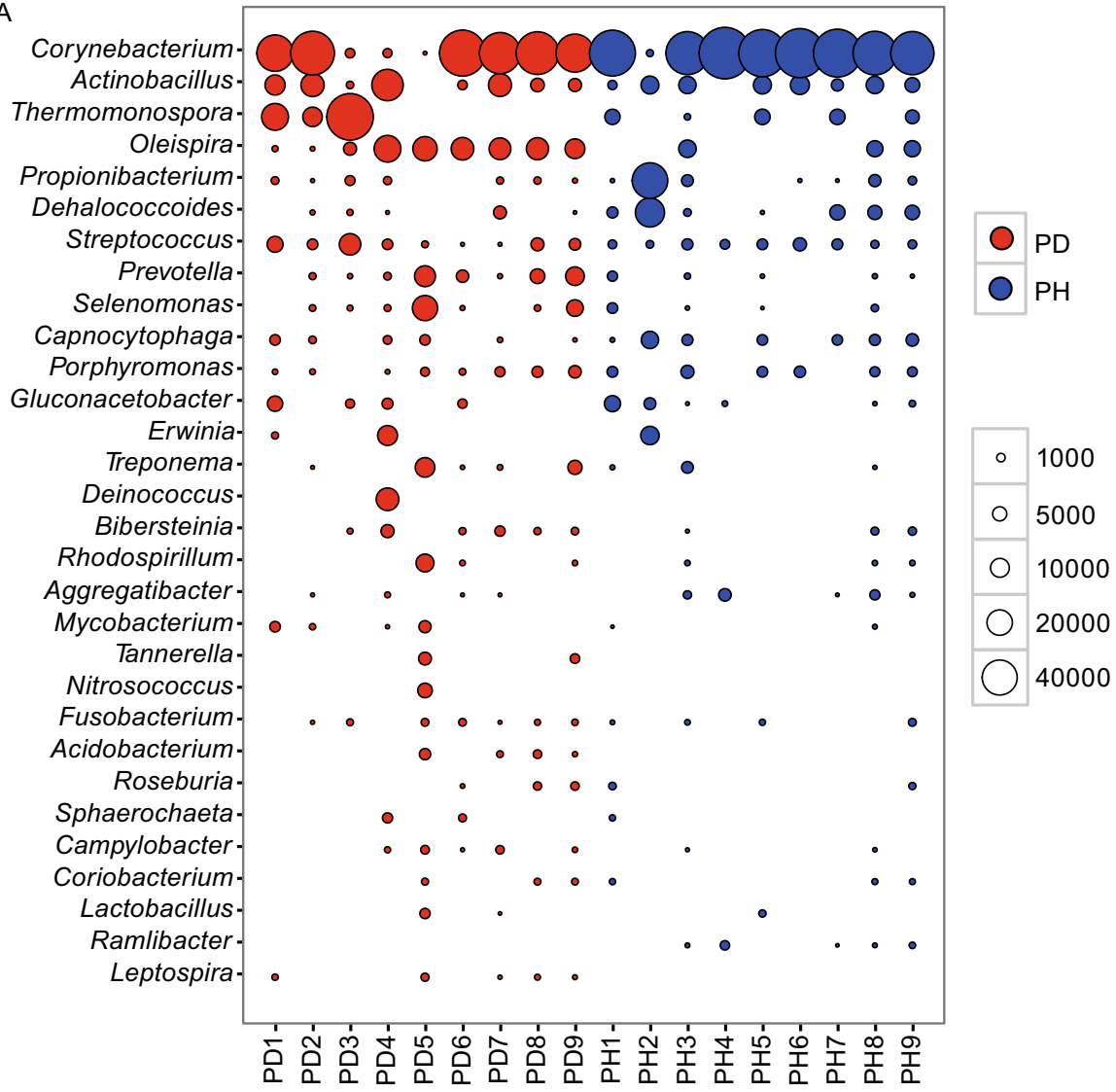

B

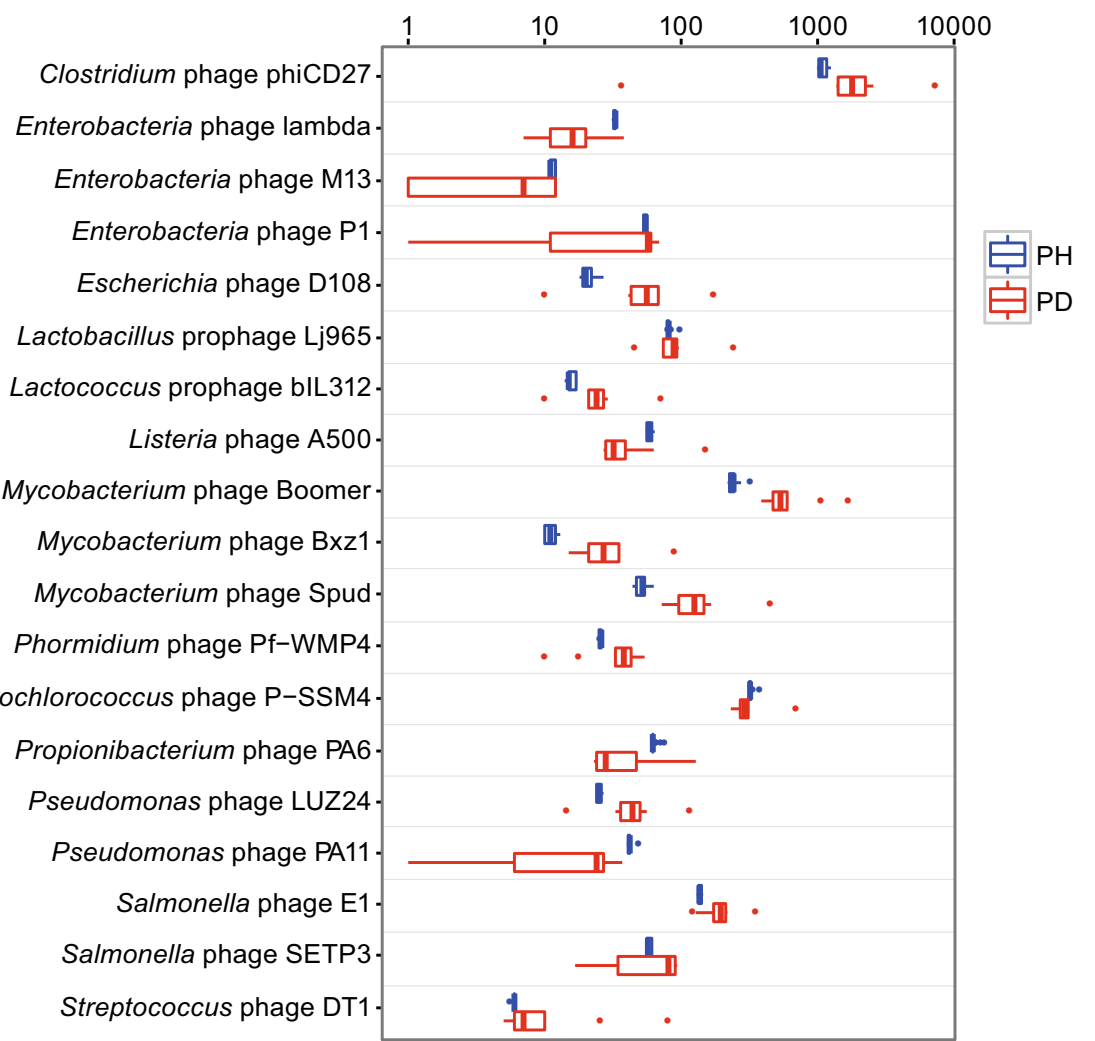


Corynebacterium, Actinobacillus, Capnocytophaga, Streptococcus and Prevotella, were all included in this consortia. However, Streptococcus and Prevotella, which usually account for the dominant of oral microbiota, are not the leading contributors of DRs. This result suggests the abundance of CRISPRs of microbial community in human oral cavities is not only determined by the amount of bacteria, but also closely related with species composition. Previous studies have reported that some bacteria were highly abundant in periodontitis, such as Prevotella, Selenomonas and Treponema (Wang et al., 2013). Porphyromonas gingivalis, Tannerella forsythia and Treponema denticola were also widely known as 'the red complex' to be involved in the periodontal diseases (Darveau, 2010). Although we found that the DR abundance of several genera (e.g., Prevotella, Selenomonas, Treponema and Tannerella) in PD samples was a little higher than that in $\mathrm{PH}$ samples, we did not observe any of them with significant difference by MannWhitney $U$ test. This reveals that the ability of defense to phages is at least not weaker in healthy people than in patients.

To further investigate the primary cause of significant differences in CRISPR elements between periodontally diseased patients and healthy people, we conducted F-test to compare the abundance variation of each DRs and spacers. We used $0.0007(0.05 / 68)$ and $0.001(0.05 / 32)$ as $P$-value cutoffs to perform the Bonferroni correction for multiple testing of DRs (68 genera) and spacers (32 genera), respectively. For 68 bacterial genera carrying DRs, abundance variation of 32 genera was significantly lower in health people than in patients (Fig. S3). We also observed a similar situation for spacers (Fig. 2B). The spacers aligned to 305 of 444 phage species had greater concordance in healthy people, and they occupied $69.2 \%$ of total spacers. 96 species were left even with $P$-value cutoff 0.0001 . The most substantial variation came from the species Clostridium phage phiCD27. We also classified these spacers to the genus level and got a similar result. 23 out of 28 genera had greater concordance in healthy people, and they occupied $91.8 \%$ of all the spacers. Since spacers indicate the ability of bacteria to resist invasion of certain bacteriophages, and thus the microbiome in healthy people seems to be fairly adapted to defend invasion of such phages and could be much more robust to micro-environment alteration.

In conclusion, by systematical analysis of CRISPR using whole genome sequencing data for oral microbiome, we found the composition of DRs and spacers are significantly different between PD and PH. The defense potentials by CRISPRs were related to both evenness and richness of oral

Huiyue Zhou and Hui Zhao have equally contributed to this work.

Electronic supplementary material The online version of this article (doi:10.1007/s13238-015-0182-0) contains supplementary material, which is available to authorized users. bacteria. Healthy people had less DRs and more spacers and were more similar with each other, which will assemble a robust and functional bacterial community to resist the invasion from phage. CRISPRs may play some roles in the equilibrium of microbial community. However, the details of how they function in the oral ecology still need more exploration.

\section{FOOTNOTES}

This work was supported by the National Natural Science Foundation of China (Grant No. 31300110) to J. Wang and (Grant No. 31301031 ) to Y. Zhang. We thank L. Hou and L. Zhang (Research Facility Center at Beijing Institutes of Life Science, Chinese Academy of Science) for their sequencing assistance.

Huiyue Zhou, Hui Zhao, Jiayong Zheng, Yuan Gao, Yanming Zhang, Fangqing Zhao and Jinfeng Wang declare that they have no conflict of interest. All procedures followed were in accordance with the ethical standards of the responsible committee on human experimentation (Beijing Institutes of Life Science, CAS) and with the Helsinki Declaration of 1975, as revised in 2000 (5). Informed consent was obtained from all patients for being included in the study.

Huiyue Zhou ${ }^{1,2}$, Hui Zhao ${ }^{3}$, Jiayong Zheng ${ }^{3}$, Yuan Gao ${ }^{1,2}$, Yanming Zhang ${ }^{1}$, Fangqing Zhao ${ }^{1}$, Jinfeng Wang ${ }^{1 凶}$

${ }^{1}$ Computational Genomics Lab, Beijing Institutes of Life Science,

Chinese Academy of Sciences, Beijing 100101, China

2 University of Chinese Academy of Sciences, Beijing 100049,

China

${ }^{3}$ Wenzhou People's Hospital, Wenzhou 325000, China

$\triangle$ Correspondence: wangjf@mail.biols.ac.cn (J. Wang)

\section{OPEN ACCESS}

This article is distributed under the terms of the Creative Commons Attribution 4.0 International License (http://creativecommons.org/ licenses/by/4.0/), which permits unrestricted use, distribution, and reproduction in any medium, provided you give appropriate credit to the original author(s) and the source, provide a link to the Creative Commons license, and indicate if changes were made.

\section{REFERENCES}

Andersson AF, Banfield JF (2008) Science 320:1047-1050

Barrangou R, Fremaux C, Deveau H, Richards M, Boyaval P, Moineau S, Romero DA, Horvath P (2007) Science 315:1709-1712

Belda-Ferre P, Alcaraz LD, Cabrera-Rubio R, Romero H, SimonSoro A, Pignatelli M, Mira A (2012) ISME J 6:46-56

Curtis MA, Zenobia C, Darveau RP (2011) Cell Host Microbe 10:302-306

Darveau RP (2010) Nat Rev Microbiol 8:481-490

Ghorbal M, Gorman M, Macpherson CR, Martins RM, Scherf A, Lopez-Rubio JJ (2014) Nat Biotechnol 32:819-821

Grissa I, Vergnaud G, Pourcel C (2007) BMC Bioinformatics 8:172 Jansen R, Embden JD, Gaastra W, Schouls LM (2002) Mol Microbiol 43:1565-1575 
Pride DT, Sun CL, Salzman J, Rao N, Loomer P, Armitage GC, Banfield JF, Relman DA (2011) Genome Res 21:126-136

Pride DT, Salzman J, Relman DA (2012) Environ Microbiol 14:25642576

Rho M, Wu YW, Tang H, Doak TG, Ye Y (2012) PLoS Genet 8: e1002441
Robles-Sikisaka R, Ly M, Boehm T, Naidu M, Salzman J, Pride DT (2013) ISME J 7:1710-1724

Skennerton CT, Imelfort M, Tyson GW (2013) Nucleic Acids Res 41:e105 Tyson GW, Banfield JF (2008) Environ Microbiol 10:200-207

Wang J, Qi J, Zhao H, He S, Zhang Y, Wei S, Zhao F (2013) Sci Rep 3:1843 